\title{
The Mean of Arithmetic Functions and the Solvability of a Class of Diophant Equations
}

\author{
Rajive Atri \\ Associate Professor and Head, Department of Mathematics, C.S.S.S. (PG) College, Machhra, Meerut, India \\ rajiveatri20@gmail.com
}

Abstract: In this paper, the above Diophantine equations have been discussed for integral solutions.

Keywords: Diophantine equation and integral solution.

\section{Introduction}

Erdos \& Straus (1950) conjectured that for all integers $n \geq 2$, the rational number $\frac{4}{n}$ can be expressed as sum three unit fractions. Thus the conjecture formally states that for every integer $n \geq 4$, there exists positive integers $x, y$ and $z$ such that $\frac{4}{n}=\frac{1}{x}+\frac{1}{y}+\frac{1}{z}$.

Monks, M. \& Velingker, A. examined the solutions of the above equation for prime $n$. Hari Kishan et. al. (2011) discussed the Diophantine equations of second and higher degree of the form $3 x y=n(x+y)$ and $3 x y z=$ $n(x y+y z+z x)$ etc. Rabago, J. F.T. \& Tagle, R.P. (1913) discussed the area and volume of a certain regular solid and the Diophantine equation $\frac{1}{2}=\frac{1}{x}+\frac{1}{y}+\frac{1}{z}$. Sander, J. (1913) discussed the Diophantine equation $\frac{1}{2}=\frac{1}{x}+\frac{1}{y}+\frac{1}{z}$ and obtained solutions of this Diophantine equation.

In this paper, the above problem has been extended for the Diophantine equations $\frac{1}{x}+\frac{1}{y}+\frac{1}{z}=\frac{1}{4}$ and $\frac{1}{x}+\frac{1}{y}+\frac{1}{z}+\frac{1}{t}=\frac{1}{4}$.

\section{A nalysis:}

Case 1: First, the Diophantine equation $\frac{1}{x}+\frac{1}{y}+\frac{1}{z}=\frac{1}{4}$ has been discussed. Suppose $x \leq y \leq z$. Then $\frac{1}{x}<\frac{1}{4}$. This implies that $x \geq 5$. Also $\frac{3}{x} \geq \frac{1}{4}$. This implies that $x \leq 12$. Thus $x=\{5,6,7,8,9,10,11,12\}$. Thus there may be the following cases:

$$
\begin{array}{ll}
\text { For } & x=5, \frac{1}{y}+\frac{1}{z}=\frac{1}{20}, \\
\text { For } & x=6, \frac{1}{y}+\frac{1}{z}=\frac{1}{12}, \\
\text { For } & x=7, \frac{1}{y}+\frac{1}{z}=\frac{3}{28}, \\
\text { For } & x=8, \frac{1}{y}+\frac{1}{z}=\frac{1}{8}, \\
\text { For } & x=9, \frac{1}{y}+\frac{1}{z}=\frac{5}{36}, \\
\text { For } & x=10, \frac{1}{y}+\frac{1}{z}=\frac{3}{20}, \\
\text { For } & x=11, \frac{1}{y}+\frac{1}{z}=\frac{7}{44}, \\
\text { For } & x=12, \frac{1}{y}+\frac{1}{z}=\frac{1}{6} .
\end{array}
$$

Equations (1), (2), (4) and (8) imply the following relations:

\begin{tabular}{|c|c|c|c|}
\hline & & & \\
\hline 0$)=1,(z$ & $20)=400 ;(y$ & $20)=2,(z$ & $20)=200 ;$ \\
\hline$)=4,(z$ & $20)=100 ;(y$ & $20)=5,(z$ & $20)=80$ \\
\hline $\begin{aligned}20) & =8,(z \\
-20) & =20,(z\end{aligned}$ & $\begin{array}{l}20)=50 ;(y \\
-20)=20\end{array}$ & $20)=10,(z$ & $20)=40$ \\
\hline
\end{tabular}
$(y \quad 20)(z \quad 20)=400$,

$$
\begin{aligned}
& (y-12)(z-12)=144 \\
& (y-8)(z-8)=64 \\
& \left(\begin{array}{ll}
y & 6
\end{array}\right)\left(\begin{array}{ll}
z & 6
\end{array}\right)=36
\end{aligned}
$$

From (9), the following cases may be possible:

Thus we have the following solutions:

$(x, y, z)=(5,21,420),(5,22,220),(5,24,120),(5,25,100)$, $(5,28,70)$,

$(5,30,60),(5,40,40)$.

From (10), the following cases may be possible:

$(y \quad 12)=1,\left(\begin{array}{ll}z & 12\end{array}\right)=144 ;\left(\begin{array}{ll}y & 12\end{array}\right)=2,\left(\begin{array}{ll}z & 12\end{array}\right)=72$;

$(y-12)=3,(z-12)=48 ;(y-12)=4,(z-12)=36$;

$(y-12)=6,(z-12)=24 ;(y-12)=8,(z-12)=16$;

$(y-12)=12,(z-12)=12$.

Thus we have the following solutions:

$(x, y, z)=(6,13,156),(6,14,84),(6,15,60),(6,16,48)$, $(6,18,36)$,

$(6,20,28),(6,24,24)$.

From (11), the following cases may be possible: $(y-8)=1,(z-8)=64 ;(y-8)=2,(z-8)=32$; $\left(\begin{array}{ll}y & 8\end{array}\right)=4,\left(\begin{array}{ll}z & 8\end{array}\right)=16 ;\left(\begin{array}{ll}y & 8\end{array}\right)=8,\left(\begin{array}{ll}z & 8\end{array}\right)=8$.

Thus we have the following solutions:

$(x, y, z)=(8,9,72),(8,10,40),(8,12,24),(8,16,16)$,

From (12), the following cases may be possible:

$(y-6)=1,(z-6)=36 ;(y-6)=2,(z-6)=18$;

$\left(\begin{array}{ll}y & 6\end{array}\right)=3,\left(\begin{array}{ll}z & 6\end{array}\right)=12 ;\left(\begin{array}{ll}y & 6\end{array}\right)=4,\left(\begin{array}{ll}z & 6\end{array}\right)=9$;

$(y-6)=6,(z-6)=6$.

Thus we have the following solutions:

$(x, y, z)=(12,7,42),(12,8,24),(12,9,18),(12,10,15)$, $(12,12,12)$.

Similarly the solutions the solutions for $x=6,7,8,9,10,11$ and 12 can be obtained. The solutions can further be obtained by using the equations (3), (5), (6) and (7). Since $x$, 
$y$ and $z$ are symmetric in the Diophantine equation there are $3 !=6$ cases for each solution.

Case 2: Second, the Diophantine equation $\frac{1}{x}+\frac{1}{y}+\frac{1}{z}+\frac{1}{t}=\frac{1}{4}$ has been discussed. Suppose $x \leq y \leq z \leq t$. Then $\frac{1}{x}<\frac{1}{4}$. This implies that $x \geq 5$. Also $\frac{4}{x} \geq \frac{1}{4}$. This implies that $x \leq 16$. Therefore $x=\{5,6,7,8,9,10,11,12,13,14,15,16\}$. Thus there may be the following cases:

$\begin{array}{ll}\text { For } & x=5, \frac{1}{y}+\frac{1}{z}+\frac{1}{t}=\frac{1}{20}, \\ \text { For } & x=6, \frac{1}{y}+\frac{1}{z}+\frac{1}{t}=\frac{1}{12}, \\ \text { For } & x=7, \frac{1}{y}+\frac{1}{z}+\frac{1}{t}=\frac{3}{28}, \\ \text { For } & x=8, \frac{1}{y}+\frac{1}{z}+\frac{1}{t}=\frac{1}{8}, \\ \text { For } & x=9, \frac{1}{y}+\frac{1}{z}+\frac{1}{t}=\frac{5}{36}, \\ \text { For } & x=10, \frac{1}{y}+\frac{1}{z}+\frac{1}{t}=\frac{3}{20}, \\ \text { For } & x=11, \frac{1}{y}+\frac{1}{z}+\frac{1}{t}=\frac{7}{44}, \\ \text { For } & x=12, \frac{1}{y}+\frac{1}{z}+\frac{1}{t}=\frac{1}{6}, \\ \text { For } & x=13, \frac{1}{y}+\frac{1}{z}+\frac{1}{t}=\frac{7}{78}, \\ \text { For } & x=14, \frac{1}{y}+\frac{1}{z}+\frac{1}{t}=\frac{2}{21}, \\ \text { For } & x=15, \frac{1}{y}+\frac{1}{z}+\frac{1}{t}=\frac{1}{10}, \\ \text { For } & x=16, \frac{1}{y}+\frac{1}{z}+\frac{1}{t}=\frac{1}{16},\end{array}$

Equation (13) gives $\frac{1}{y}<\frac{1}{20}$. This implies that $y \geq 21$. Also $\frac{3}{y} \geq \frac{1}{20}$. This implies that $y \leq 60$. Therefore $y=$ $\{21,22,23, \ldots, 60\}$. Thus there may be the following cases:

$$
\begin{aligned}
& \text { For } y=21, \frac{1}{z}+\frac{1}{t}=\frac{1}{420}, \\
& \text { For } y=22, \frac{1}{z}+\frac{1}{t}=\frac{1}{220}, \\
& \text { For } y=23, \frac{1}{z}+\frac{1}{t}=\frac{3}{460}, \\
& \text { For } y=24, \frac{1}{z}+\frac{1}{t}=\frac{1}{120}, \\
& \text { For } y=28, \frac{1}{z}+\frac{1}{t}=\frac{1}{70}, \\
& \text { For } y=32, \frac{1}{z}+\frac{1}{t}=\frac{3}{160}, \\
& \text { For } y=40, \frac{1}{z}+\frac{1}{t}=\frac{1}{40}, \\
& \text { For } y=56, \frac{1}{z}+\frac{1}{t}=\frac{9}{280}, \\
& \text { For } y=60, \frac{1}{z}+\frac{1}{t}=\frac{1}{30},
\end{aligned}
$$

Equations (25), (26), (28), (29), (31) and (33) imply the following relations:

$$
\begin{aligned}
& (z-420)(t-420)=176400 \\
& (z-220)(t-220)=48400 \\
& (z-120)(t-120)=14400 \\
& (z-70)(t-70)=4900 \\
& (z-40)(t-40)=1600 \\
& (z-30)(t-30)=900
\end{aligned}
$$

From equation (37), the following cases may be possible: $(z-70)=1,(t-70)=4900 ;(z-70)=2,(t-70)=2450$; $(z-70)=4,(t-70)=1225 ;(z-70)=5,(t-70)=980$; $(z-70)=7,(t-70)=700 ;(z-70)=10,(t-70)=490 ;$ $(z-70)=14,(t-70)=350 ;(z-70)=20,(t-70)=245 ;$ $(z-70)=28,(t-70)=175 ;(z-70)=35,(t-70)=140 ;$ $(z-70)=49,(t-70)=100 ;(z-70)=70,(t-70)=70$.

Thus we have the following solutions:

$$
\begin{gathered}
(x, y, z, t)=(5,28,71,4970),(5,28,72,2520),(5,28,74,1295), \\
(5,28,75,1050),(5,28,77,770),(5,28,80,560),(5,28,84,420),(5,28,90,325) \\
,(5,28,98,245),(5,28,105,210),(5,28,119,170),(5,28,140,140) .
\end{gathered}
$$

Similarly the other solutions can be obtained for other values of $x$ and $y$ and for relations (25), (26), (29), (31) and (33). Since $x, y, z$ and tare symmetric in the Diophantine equation there are $4 !=24$ cases for each solution.

\section{Concluding Remarks}

Here the two Diophantine equations have been discussed for integral solutions for certain values of variables $x$ and $y$. More solutions can further be obtained for other values of variables $x$ and $y$ and for other relations in the variables.

\section{References}

[1] Guy, R.K. (1994): Unsolved Problems in Number Theory. Springer, Verlag, New Yark

[2] Kishan, H., Rani, M. and Agarwal, S. (2011): The Diophantine Equations of Second and Higher Degree of the form $3 x y=n(x+y)$ and $3 x y z=n(x y+y z+$ $z x$ etc. Asian Journal of Algebra, 4(1), 31-37.

[3] Rabago, J. F.T. \& Tagle, R.P.(2013): The area and volume of a certain regular solid and the Diophantine equation $\frac{1}{2}=\frac{1}{x}+\frac{1}{y}+\frac{1}{z}$. Notes on Number Theory and Discrete Mathematics, 19(3), 28-32.

[4] Sander, J. (1913): A Note on a Diophantine equation. Notes on Number Theory and Discrete Mathematics, 19(4), 1-3.

[5] Erdos, P. \& Straus, E.G. (1950): On a Diophantine Equation. Math. Lapok, 1, 192-210. 